\author{
Nilam Khadka, MCh \\ Neurosurgical services \\ National Trauma Centre, \\ National Academy of Medical Sciences (NAMS) \\ Kathmandu, Nepal.
}

Rajan Kumar Sharma, MCh Resident

Department of Neurosurgery

Bir Hospital, NAMS

Kathmandu, Nepal.

Rajiv Jha, MCh

Department of Neurosurgery

Bir Hospital, NAMS

Kathmandu, Nepal.

Prakash Bista, MCh

Department of Neurosurgery

Bir Hospital, NAMS

Kathmandu, Nepal.

\author{
Address for correspondence: \\ Dr Nilam Khadka, MCh \\ Neurological Services, \\ National Trauma Centre, NAMS \\ Kathmandu, Nepal. \\ Email: nilambins@gmail.com \\ Phone No. +977 1 4226634/4239161
}

Date submitted: $16 / 7 / 2018$

Date accepted: $25 / 8 / 2018$

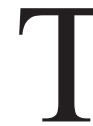

he primary aim of the intensive care management of traumatic brain injury (TBI) is to prevent and treat secondary ischemic injury using a multifaceted neuroprotective strategy to maintain cerebral

\section{Study of Intracranial Pressure Monitoring In Traumatic Brain Injury}

Intracranial pressure monitoring is considered the standard of care for severe traumatic brain injury and is used frequently.However, the efficacy of treatment based on monitoring in improving the outcome has not been rigorously assessed.

We conducted a trial in which we included 26 patients of all types of traumatic brain injury (TBI) and they were monitored for intracranial pressure by Conventional fluid filled systemwith a manometer (Group1) and compared with the Fibreoptictransducer-tipped intracranial pressure monitoring system(Group 2).The main aim of this study was to examine the relationship between Intracranial Pressure (ICP) monitoring and in-hospital mortality.

The median length of stay in the ICU was similar in the two groups (12 days in the conventional pressure-monitoring group and 9 days in the new fibreoptic group; $\mathrm{P}=0.25$ ), the number of days of brain-specific treatments (e.g., administration of hyperosmolar fluids and the use of hyperventilation) in the ICU was similar in both groups. The distribution of serious adverse events was similar in the two groups.

We concluded that ICP monitoring (as is any monitoring modality) is a useful guide for management. The outcomes are decided by the differences in management protocols that the knowledge of the said parameter brings about. ICP monitoring is recommended for the better management of traumatic brain injury and fibreoptic ICP monitoring seems to be beneficial than using the conventional methods of ICP monitoring with manometer.

Key words: Brain Trauma foundation, Camino, Fibreoptic intracranial monitor, ICP, TBI, Nepal

perfusion to meet the brain's metabolic demands for oxygen and glucose. Because the brain is encased by the nonexpendable skull, an increase in intracranial pressure (ICP) may impede cerebral blood flow (CBF) and lead to 


\section{Department of Neurosurgery, NAMS, Bir Hospital, Kathmandu.}

\section{Protocol for Intracranial Pressure (ICP) Measurement}

\section{Indications for ICP monitoring}

- Severe diffuse head injury - extending to pain or worse

- Postoperatively in all traumatic intradural mass lesions

- Extradural haematomas with preoperative dilated pupil

- Intracranial haematoma of borderline size managed conservatively

- Polytrauma patients requiring prolonged ventilation

- Following major elective intracranial surgery

\section{Duration}

- Continue until the ICP has remained normal for 48 hours

- Resitation of the ventriculostomy catheter after 5 days if monitoring is required for a prolonged period.

\section{Please make sure}

- The transducer is kept at the level of the forehead.

- Zero calibration is done every 4 hours

- The doctor is informed for appropriate action if the ICP remains over $25 \mathrm{~mm}$ of $\mathrm{Hg}$ for more than 15 minutes

- The ICP catheters and connections are handled with utmost sterile precautions.

Prof. P. Kumar

Chief \& Consultant Neurosurgeon

Figure 1: Departmental Protocol of ICP Measurement.

cerebral ischemia. Increased ICP is an important cause of secondary brain injury, and its degree and duration is associated with outcome after TBI. ${ }^{16,15}$ ICP monitoring is the most widely used intracranial monitor because prevention and control of increased ICP and maintenance of cerebral perfusion pressure (CPP) are fundamental therapeutic goals after TBI.

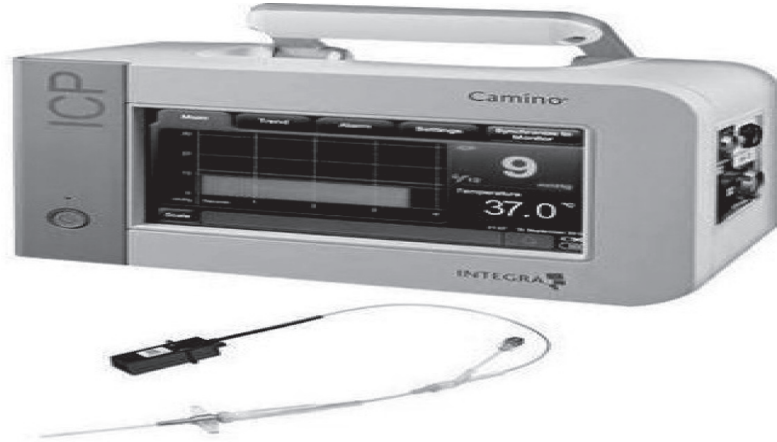

Figure 2: Camino Fibreoptic ICP monitor

Traumatic brain injury (TBI) remains a major cause of deathand disability worldwide. In the United States alone, morethan 53,000 individuals die annually because of TBI, contributingto $30.5 \%$ of all injury-related deaths. In 2010, the estimated burdenof TBI on the U.S. economy was approximately $\$ 76.5$ billion.After the initial injury, mass lesions, an increase in brain-water content (edema), and an increase in blood volume, can result inrising pressure in the rigid skull, which may lead to brain tissueherniation, impaired cerebral perfusion, and, without intervention, further damage to the brain. Among those who die from TBI, themajority die because of uncontrolled rise of intracranial pressure(ICP), mostly within the first 48 hours of injury. After a severe TBI, efforts are focused on prevention of further damage through intensive monitoring and prompt intervention. ${ }^{11}$ In 1951, Guillaume and Janny first described continuous ICP monitoring using an electronic magnetic transducer to measure changes in ventricular fluid pressure. Since then, invasive ICP monitoring has become an increasingly employed tool to care for patients with severe TBI and has been adopted as part of the Brain Trauma Foundation (BTF) guidelines for the management of severe TBI, based on indirect evidence in which observational studies linked increasedICP with worse outcomes. ${ }^{19,3}$

\section{Materials and Methods:}

This is a prospective study. Weconducted the study of first series of patients during 2011-2016in which we included 26 patients of all types of traumatic brain injury and they were monitored for intracranial pressure by the conventional method of ICP monitoring with the fluid filled catheter system with manometer (Group 1).For this, we followed the existing protocol of ICP monitoring of the Department of Neurosurgery of Bir Hospital (Figure1). The catheters were placed either intraventricular or in the subdural space and the catheter was connected to a manometer (external transducer). In this group, ICP monitoring was also done in all types of traumatic 


\section{Severity of HI}
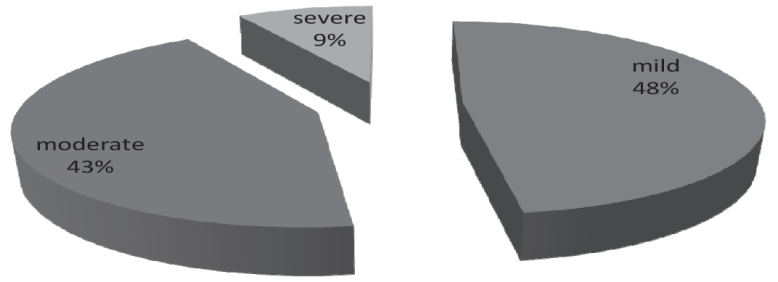

Figure 3: Severity of TBI in (Group 1) patients with Conventional method of ICP monitoring.

brain injuries including mild and moderate TBI whose radiology and neurological assessment findings indicate need of monitoring in those with intracranial hematoma or severe diffuse traumatic brain injury extending or worse. They were being treated in intensive care units (ICUs) inBir Hospital. From 2016/17, westarted using the Camino Fibreoptic Intracranial Pressure Monitoring kit (Integra) and have included 15 patients of severe TBI in this group (Group 2) and have compared with the conventional methods of Intracranial Pressure monitoring (Figure 2). For indications of ICP monitoring for this group of patients, we followed the Brain Trauma Foundation guidelines $2007^{3}$-firstly, all salvageable patients with a TBI (GCS 3-8 after resuscitation) and an abnormal CT scan. (An abnormal CT scan of the head is one that reveals hematomas, contusions, swelling, herniation, or compressed basal cisterns). Secondly, allthe patients with severe TBI with a normal CT scan if two or more of the following features are noted at admission: age over 40 years, unilateral or bilateral motor posturing, or systolic blood pressure (BP)<90 mm Hg. The Camino fibreoptic monitoring kit contains a sterile transducer-tipped pressure monitoring catheter and its method of measuring pressure is unique.

This was an observational cohort study, with the exposure of interest being invasive ICP monitoring in patients with severe TBI. The main aim of this study was to examine the relationship between ICP monitoring and in-hospital mortality.

We used two analytic approaches to assess this relationship. First, we used ICP monitoring as a patientlevel variable to determine the association between ICP monitoring and mortality. Previously, we were using the conventional method of fluid filled catheter system for the ICP monitoring.Secondly, after the availability of the Caminofibreoptic ICP monitoring system in National Trauma Centre, we started to use it solely as it has ease of insertion, can be inserted in severely compromised

\section{Types of ICP Catheters}

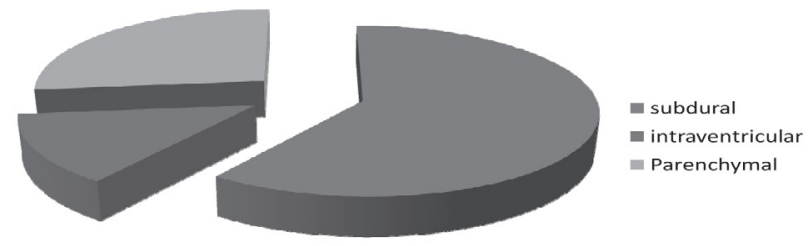

Figure 4: Sites of catheter insertion in (Group 2) patients with Fibreopticcatheter as ICP Monitoring.

ventricles or those with midline shift, has low risk of hemorrhage and infection and has zero drift. The subdural fibreoptic catheters were used in those patients who underwenteithercraniotomyordecompressivecraniectomy. The parenchymal catheters were used mainly in those patients with diffuse brain injuries or who did not need craniotomy or craniectomy as the initial part of treatment. The intraventricular catheters were used in those patients who had normal sized ventricles. We compared this method with the conventional methodof ICP monitoring; however, we only recruited the severe TBI patients in Group 2.

We used data derived from the medical record departments of Bir Hospital and National Trauma Centre,both hospitals belonging to the National Academy of Medical Sciences (NAMS). All detailed history and the physical examination were recorded and all the required data were taken for the study.The primary endpoint for this study was the odds of in-hospital death after TBI.

\section{Statistical analysis}

The primary hypothesis was tested with the use of the blocked Wilcoxon test, with blocking on stratification factors, and a two-sided significance level of 0.05. We obtained odds ratios and confidence intervals from a logistic proportional-odds model, accounting for the same factors. This analysis was supplemented by similar analyses of individual measures and composite analyses of subgroup measures. Cox models were used to analyze survival. A significance level of 0.01 was used to test secondary hypotheses. The main analyses included data on all participants randomly assigned to a treatment group (intention-to-treat population). Sensitivity analyses included analyses restricted to patients who survived.

We calculated standardized differences to compare baseline characteristics between those who underwent ICP monitoring by conventional method and the new method of fibreoptic ICP monitoring. 


\section{Khadka et al}

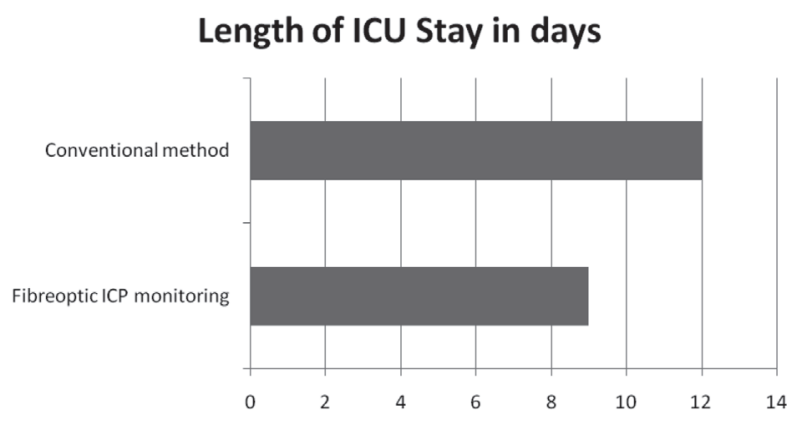

Figure 5: Comparing length of ICU stay in Conventional method (Group 1) with Fibreoptic method (Group 2).

\section{Results:}

The mean age of presentation for the patients who underwent ICP Monitoring by using the conventional methodwas 52 years. There were total of 26 patients who were included in the study for the ICP monitoring by the conventional method. The maximum patients were in age group of 40 to 60 years which were 13 . The mode of injury was fall in maximum number of patients (11) followed by seven patients of Road traffic accident.Almost half of the patients $(48 \%)$ had mild head injury followed by moderate head injury (43\%) and severe head injury (9\%) respectively (Figure 3). After monitoring the ICP of these patients good recovery was seen in 8 patients and we had 8 mortality in the series of 26 patients whom we used the conventional method as the ICP monitoring tool. ICP monitoring was also helpful in non-severe traumatic brain injury case, too from old cohort (Group 1) as one of the cases had to undergo evacuation of hematoma as the ICP started to rise and subsequent CT scan of the patient showed expanding hematoma.

We have now started using the Camino fibreoptic monitoring system for the ICP monitoring and have included 15 patients in the series (Group 2). The mean age of presentation for the patients who underwent ICP Monitoring by using Fibreoptictransducers was 48 years. The maximumnumbers of patients were in age groups of 20 to 40 years and there were five patients of the age more than 60 years. There were eight patients of fall followed by three patients of Road traffic accident. We placed the catheter in subdural space in nine patients andintraventricular in two patientsandparenchymal in four patients (Figure 4). Reason of using suduralcatheters in majority of cases is for its ease of placementduring the craniotomy or craniectomy who needed it and its inherent less complications. We have included only the severe TBI patients in the Fibreoptic monitoring series.After monitoring the ICP of the all the 15 patients of severe

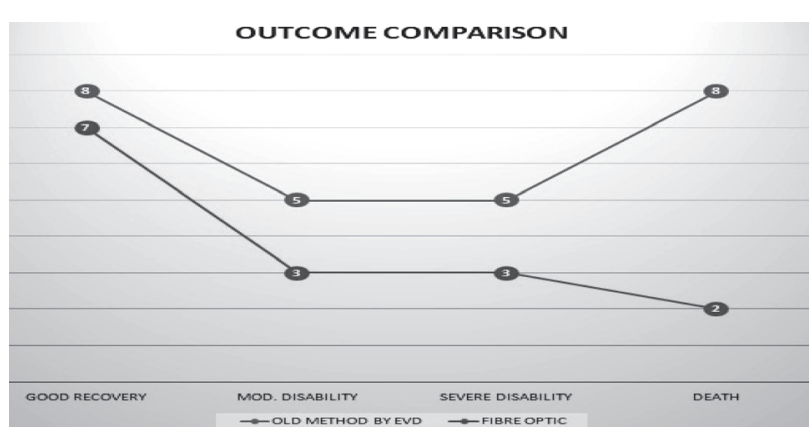

Figure 6: Comparing outcome of patients in the conventional method (Group 1) with Fibreoptic method (Group 2).

TBIin this series (Group 2), good recovery was seen inseven patients and we hadtwo deaths.

There were significant differences between the conventional method of Intracranial Pressure Monitoring and the new fibreoptic method. The good recovery was seen in almost half of the patients with new fibreoptic method of Intracranialpressure monitoring compared to the conventional method. The median length of stay in the Intensive Care Unit (ICU) was similar in the two groups (12 days in the conventional pressure-monitoring group and 9 days in the new fibreopticgroup; $\mathrm{P}=0.25$ ) (Figure 5), the number of days of brain-specific treatments (e.g., administration of hyperosmolar fluids and the use of hyperventilation) in the ICU was similar in both groups. The distribution of serious adverse events was similar in the two groups. There was significant reduction in the mortality in the Group-2 patients (eight mortality out of 26 patientsin the conventionalmethod and only two mortality in the new fibreoptic method; $p=<0.01$ ) (Figure 6). The reason can be due to the good neurocriticaland nursing care by health personnel in National Trauma Centre.The old cohort (Group 1) had high chances of malposition, occlusion, infection and hemorrhage compared to the new cohort (Group 2) ofFibreoptic ICP monitoring.

\section{Discussion}

In the field of neurotrauma and critical care, invasive ICP monitoring has long been considered a standard of care for severe TBI patients without being supported by rigorous assessment of its

effectiveness in improving outcomes. Normal and pathologic ICP: Normal ICP varies with age, body position, and clinical condition. ${ }^{1}$ The normal ICP is $7-15 \mathrm{~mm} \mathrm{Hg}$ in a supine adult, 3-7 $\mathrm{mm} \mathrm{Hg}$ in children, and 1.5-6 mm Hg in term infants. The definition of intracranial hypertension depends on the specific pathology and age, although ICP $>15 \mathrm{~mm} \mathrm{Hg}$ is generally considered to be abnormal. 
In the injured brain, there may be intra parenchymal pressure gradients between the supra and infra-tentorial compartments and bilateral monitoring has revealed differential pressures across the midline in the presence of hematomas and also in the absence of space-occupying lesions. ${ }^{17}$ Increased ICP causes a critical reduction in CPP and $\mathrm{CBF}$ and may lead to secondary ischemic cerebral injury. ${ }^{28,21} \mathrm{~A}$ number of studies have shown that high ICP is strongly associated with poor outcome, particularly if the period of intracranial hypertension is prolonged. Increased ICP can also cause actual shift of brain substance resulting in structural damage to the brain and to herniation through the tentorial hiatus or foramen magnum. The latter results in pressure on the brainstem causing bradycardia and hypertension (the classic Cushing reflex) and, if untreated, respiratory depression and death. ${ }^{4,18}$

\section{General indications for ICP monitoring:}

Despite the widespread applications of ICP monitoring, there are no data from randomized controlled trials that can clarify its role in acute coma. ${ }^{27}$ With the exception of monitoring after severe TBI, the indications for ICP monitoring are not well established and vary from center to center. Case-mix adjusted mortality in comatose patients with intracranial hemorrhage is lower in those who receive ICP monitoring compared with those who do not and increased ICP is associated with poor prognosis after subarachnoid hemorrhage. ${ }^{13}$ There is little evidence to support ICP monitoring in other neurological conditions, such as acute stroke, when there is no benefit over clinical monitoring alone. ICP monitoring after anoxic injury after cardiac arrest has little value in targeting treatment, although it may be useful in hepatic encephalopathy. ${ }^{22}$

\section{ICP monitoring after TBI:}

In 1982, Narayan et al. demonstrated in a prospective study of 133 patients that outcome prediction after TBI was increased when ICP monitoring was added to standard clinical observations. Subsequently, analysis of the National Traumatic Coma Data Bank showed that the proportion of hourly ICP recordings more than $20 \mathrm{~mm} \mathrm{Hg}$ was the next most significant predictor of poor outcome after the usual clinical descriptors of age, admission motor score, and abnormal pupil responses. Despite the absence of Class 1 evidence demonstrating the benefit of ICP monitoring on outcome after TBI, there is a large body of clinical evidence supporting its use to guide therapeutic interventions, detect intracranial mass lesions early, and assess prognosis. ICP monitoring is recommended by consensus guidelines for head injury management and is accepted as a relatively low-risk, high-yield and value for money intervention. ${ }^{15,16}$
The Brain Trauma Foundation recommends ICP monitoring in all patients with a severe TBI (Glasgow Coma Score 3-8) and either an abnormal CT scan or a normal scan and the presence of two or more of thefollowing three risk factors at admission: age $>40$ years; unilateral or bilateral motor posturing; a systolic a $\mathrm{BP}<90$ $\mathrm{mm} \mathrm{Hg}$. There is around $60 \%$ chance of increased ICP in these patients. Much information is available from ICP monitoring in addition to the measurement and display of absolute ICP. CPP is easily calculated as the difference between mean ABP (MAP) and ICP (CPP =MAP - ICP) and is a measure of the pressure gradient across the cerebral vascular bed. Pathologic ICP wave forms can be identified and analyzed. ${ }^{17}$

ICP monitoring can also be augmented by measurement of indices describing cerebrovascular pressure reactivity (CVR) and pressure-volume compensatory reserve.

In the Rescue ICP Trial, the third tier of treatment consisted of decompressivecraniectomy for refractory intracranial hypertension in TBI. There have been limited studies in TBI from Nepal where ICP monitoring has been studied, whereas other studies e.g. Roka et al, in their article of Non decompressive single stage bilateral craniotomy in TBI, pointed out as one of the limitations of their study to be unavailability of ICP monitoring in their patients. $^{28}$

In our study, we demonstrated a strong association between ICP monitoring and a lower risk of death after severe TBI. This finding was consistent when we examined the effectiveness of this technology at either the patient or hospital level ${ }^{18}$ Further, there appeared to be a doseresponse, with higher rates of ICP monitoring associated with lower rates of mortality, lending further credence to a causal relationship. Accurate, continuous ICP monitoring by an invasive tool can lead to the prompt recognition of spiking pressure around the injured parts of the brain. Such recognition could potentially lead to timely intervention that is able to control the rising pressure inside the rigid skull, a process that is thought to be the leading cause of death among severe TBI victims, especially during the first 48 hours after injury. ${ }^{5,6,14}$ Despite the plausibility of its efficacy in guiding us to provide better care, many have questioned its effectiveness based on several studies that failed to provide conclusive, consistent results. This inconsistency among previous studies might explain the wide variability in ICP monitoring utilization across different hospitals. Our findings agree with a number of previous studies that support the value of ICP monitoring in TBI, but contrast with several other studies that either failed to show an association between ICP monitoring and better outcomes, or showed an association between ICP monitoring and higher mortality. ${ }^{20,26}$ 


\section{Khadka et al}

Theonly randomized trial in this area showed no difference in the primaryoutcome, a compositemeasure based on performance across 21 measures of functional and cognitive status, between care focused onmaintaining ICP at $20 \mathrm{mmHg}$ or less and care based on imaging andclinical examination in the setting of the developing world, whereICP monitoring is very rarely used. However, the trial was notsufficiently powered to detect a mortality difference between bothgroups. In addition, differences in injury characteristics, prehospital,intensive care unit (ICU) and post-ICU structure and processesof care, and the observation of "delayed mortality" due tomedical complications, accounting for more than one third of deathssubsequent to TBI in Latin America, may render as questionable anyextrapolation of epidemiologic or treatment studies from the developingto developed world. ${ }^{6,8,23}$

Several single-center and multicenter observational studies have shown that ICP-targeted management of TBI is associated with worse outcomes, including prolonged mechanical ventilation, worse functional status, and higher risk of pneumonia, acute kidney injury, and mortality. However, the results of these studies may be biased because they were retrospective and sicker patients may be more likely to receive ICP monitoring. Chesnut and colleagues conducted a prospective randomized clinical trial to determine whether ICP monitoring improves outcomes. Since invasive ICP monitoring is considered standard of care, this study would have lacked clinical equipoise if conducted in the United States. Therefore, this study had to be conducted in centers that deviated from this 'standard'. The authors recruited patients from Bolivia and Ecuador, where ICP monitoring was not the standard of care. The authors concluded that ICP guided care was not superior to care based on imagingand clinical exam alone, contrary to published guidelines..$^{9,12,24}$

The Camino fibreoptic ICP monitoring system eliminates the problems inherent in the conventional fluid-filled system of ICP monitoring. For example, it gives a precise pressure measurement and artifact free high fidelity waveforms. Moreover, it has zero drift. So, a single zero-calibration is enough for the whole period of ICP monitoring. In contrast, conventional system has to be zero calibrated every four hours. So, it is alabour intensive and has high chances of occlusion, malposition and infection. The limiting factor for the use of the Camino fibreoptic kit is its high cost. It is about five times costlier than the conventional system of ICP monitoring.

In our study, we found considerable unexplained variation inhospital mortality, even after accounting for measured patient- andhospital-level characteristics. Moreover, our data were derived from National trauma centers that have interest in high-quality careby virtue of their participation and quality improvementactivities. For that reason, study may have lower mortalityrates.

\section{Limitations Of The Study:}

This study is a single institutional study though two hospitals and therefore may not be fully generalized. Group 1 had all types of traumatic brain injuries while group 2 had only severe traumatic brain injuries. Therefore, the comparison cohort group may not be too ideal. Moreover, our sample size was small in both groups. We could not include all the patients in Group 1 who were eligible for ICP monitoring according to the Departmental Protocol for ICP measurement. Similar was the case for Group 2 patients and one of the factors was the high cost of the Fibreoptic catheters and there were various other factors, too for both the Groups. Therefore, potential introduction of errors due to small sample size cannot be ruled out.

\section{Conclusions:}

We concluded that ICP monitoring (as is any monitoring modality) is a useful guide for management. The outcomes are decided by the differences in management protocols that the knowledge of the said parameter brings about. ICP monitoring is recommended for the better management of traumatic brain injury injury and fibreoptic ICP monitoring seems to be beneficial than using the conventional method of ICP monitoring with manometer.

\section{References:}

1. Albeck MJ, Skak C, Nielsen PR, Olsen KS, Borgesen SE, Gjerris F. Age dependency of resistance to cerebrospinal fluid outflow. J Neurosurg 89:275-8, 1998

2. Balestreri M, Czosnyka M, Steiner LA, Schmidt E, Smielewski P, Matta B. Intracranial hypertension: what additional information can be derived from ICP waveform after head injury?.Acta Neurochir 146:131-4, 2004

3. Brain Trauma Foundation; American Association of Neurological Surgeons; Congress of Neurological Surgeons: Guidelines for the management of severe traumatic brain injury. J Neurotrauma 24:S1-S106, 2007

4. Chambers IR, Kane PJ, Signorini DF, Jenkins A, Mendelow AD. Bilateral ICP monitoring: its importance in detecting the severity of secondary insults. Acta Neurochir Suppl 71:42-3, 1998

5. Chesnut, RM. Intracranial pressure monitoring in brain injured patients is associated with worsening of survival. J. Trauma 65: 500-501, 2008 
6. ChesnutRM, Celix JM, Chaddock K, Dikmen S, Lujan S, Petroni, G. Outcome from severe traumatic brain in Latin America: results from the Latin American pilot traumatic coma databank. J. Neurotrauma 28: A111-A112, 2011

7. Citerio G, Stocchetti N. Intracranial pressure and outcome in severe traumatic brain injury: the quest for evidence continues. Intensive Care Med 34:1173-1174, 2008

8. Colantonio A, Escobar MD, Chipman M, Austin PC, Mirabella G, Ratcliff G .Predictors of postacute mortality following traumatic brain injury in a seriously injured population. J. Trauma 64: 876882,2008

9. Cremer OL, van Dijk GW, van Wensen E, Brekelmans GJ, Moons KG, Leenen LP, Kalkman CJ. Effect of intracranial pressure monitoring and targeted intensive care on functional outcome after severe head injury. Crit Care Med 33:2207-2213, 2005

10. Czosnyka M, Pickard JD. Monitoring and interpretation of intracranial pressure. J Neurol Neurosurg Psychiatry 75:813-21,2004

11. Finkelstein E, Corso P, Miller T. The Incidence and Economic Burden of Injuries in the United States. New York (NY): Oxford University Press; 2006.

12. Griesdale DE, McEwen J, Kurth T, Chittock DR. External ventricular drains and mortality in patients with severe traumatic brain injury. Can J NeurolSci 37:43-48, 2010

13. Heuer GG, Smith MJ, Elliott JP, Winn HR, LeRoux PD. Relationship between intracranial pressure and other clinical variables in patients with aneurysmal subarachnoid hemorrhage. J Neurosurg 101:40816,2004

14. Maas AI, Schoute JW, Stocchetti N, Bullock R, Ghajar J. Questioning the value of intracranial pressure (ICP) monitoring in patients with brain injuries. J. Trauma 65:966-967, 2008

15. Marmarou A. Increased intracranial pressure in head injury and influence of blood volume. J Neurotrauma 9:S327-32, 1992

16. Marmarou A, Anderson RL, Ward JD, Choi SC, Young HF, Eisenberg HM. Impact of ICP instability and hypotension on outcome in patients with severe head trauma. J Neurosurg 75:S59-66, 1991
17. Mazzola CA, Adelson PD. Critical care management of head trauma in children. Crit CareMed 30:S393401, 2002

18. Mindermann T, Gratzl O. Interhemispheric pressure gradients in severe head trauma in humans. ActaNeurochirSuppl 71:56-8, 1998

19. Narayan RK, Greenberg RP, Miller JD, Enas GG, Choi SC, Kishore PR. Improved confidence of outcome prediction in severe head injury. J Neurosurg 54:751-762,1981

20. Pazzaglia P, Frank G, Frank F, Gaist G. Clinical course and prognosis of acute post-traumatic coma. J. Neurol. Neurosurg. Psychiatry 38: 149-154,1975

21. Rosenwasser RH, Kleiner LI, Krzeminski JP, Buchheit WA. Intracranial pressure monitoring in the posterior fossa: a preliminary report. J Neurosurg 71:503-5, 1989

22. Schwab S, Aschoff A, Spranger M, Albert F, Hacke $\mathrm{W}$. The value of intracranial pressure monitoring in acute hemispheric stroke.Neurology 47:393-8,1996

23. Stein SC, Georgoff P, Meghan S, Mirzam KL, Falaky OM. Relationship of aggressive monitoring and treatment to improved outcomes in severe traumatic brain injury. J. Neurosurg 112: 1105-1112, 2010

24. Shafi S, Diaz-Arrastia R, Madden C, Gentilello L: Intracranial pressure monitoring in brain-injured patients is associated with worsening of survival. J Trauma 64:335-340, 2008

25. Turgeon AF, Lauzier F, Simard JF, Scales DC, Burns KE, Moore L.Mortality associated with withdrawal of life-sustaining therapy for patients with severe traumatic brain injury: a Canadian multicentre cohort study. CMAJ 183: 1581-1588, 2011

26. Valentin A, Lang T, Karnik R, Ammerer HP, Ploder J, Slany J. Intracranial pressure monitoring and case mix-adjusted mortality in intracranial hemorrhage. Crit Care Med 31:1539-42, 2003

27. Wolfla CE, Luerssen TG, Bowman RM. Regional brain tissue pressure gradients created by expanding extradural temporal mass lesion. J. Neurosurg. 86:505-10, 1997

28. Y.B. Roka, A.J. Thapa. Non decompressive single stage bilateral craniotomy in traumatic brain injury. Nepal journal of Neuroscience 10.2: 45-48, 2013 